\title{
Occurrence of Pesticides in Pericarpium Citri Reticulatae and Related Products Using Syringe Filter-Based Cleanup
}

\author{
Fuxin Liu, ${ }^{a, \#}$ Xiaowen Dou, ${ }^{b, \#}$ Jiaoyang Luo, ${ }^{b}$ Dandan Kong, ${ }^{b}$ Zhuowen Fan ${ }^{*, a}$ and \\ Meihua Yang*,b \\ ${ }^{a}$ College of Pharmacy, Heilongjiang University of Chinese Medicine, 150040 Harbin, Heilongjiang, China \\ ${ }^{b}$ Key Laboratory of Bioactive Substances and Resources Utilization of Chinese Herbal Medicine, \\ Ministry of Education, Institute of Medicinal Plant Development, Chinese Academy of Medical Sciences, \\ Peking Union Medical College, 100193 Beijing, China
}

\begin{abstract}
The study established a method of rapid cleanup using an easy to operate syringe filter and gas chromatography coupled to an electron capture detector (GC-ECD) to detect 37 pesticides in Pericarpium Citri Reticulatae and related products. The critical parameters related to clean-up efficiency were optimized. The adsorbents, which included PestiCarb $(0.5 \mathrm{~g})$, primary secondary amine (PSA, $0.25 \mathrm{~g}$ ) and Florisil (1.0 g), were loaded in turn and push-pull was performed 4 times within a 1 min operating time. Under optimized conditions, the recovery of pesticides ranged from 61.6 to $128.6 \%$ at three spiked levels $\left(25,50,500 \mu \mathrm{g} \mathrm{kg}^{-1}\right)$. After analysis by GC-ECD and confirmation by gas chromatography coupled to tandem mass spectrometry (GC-MS/MS), 8 out of 57 batches of peels were found to be contaminated with hexachlorobenzene, dicofol, procymidone and p,p'-DDE (dichlorodiphenyldichloroethylene). The most frequently (10.5\%) occurring pollutants were hexachlorobenzene and dicofol. In addition, 5 batches contained prohibited or restricted persistent organochlorines at levels above regulations, and 5 of these peels contained more than two pesticides.
\end{abstract} cleanup

Keywords: Pericarpium Citri Reticulatae, GC-ECD, multiple pesticides, syringe filter-based

\section{Introduction}

Pericarpium Citri Reticulatae ("Chenpi” in Chinese) is the dried peel of ripen fruit of plant Citrus reticulata Blanco and its cultivars. The plant-originated herb has been consumed around the world for centuries as dietary supplements, condiments, snack foods, and medicinal teas due to their high nutritional, medical and energetic value. ${ }^{1-4}$ Many consumers use plant-originated products with the assumption that "natural means safe", which is not necessarily true. A pesticide residues survey in citrus fruit from Geneva indicated $86 \%$ of samples contained pesticides, and some residues stayed a high percentage in the peel. ${ }^{5}$ Our previous study also showed the tangerine peels were contaminated with multiresidues. ${ }^{6}$ The citrus plant and fruit are prone to pest infection such as pathogens, mites and aphids, insecticides are heavily used for field, plant and

*e-mail: 1282776014@qq.com; yangmeihua15@hotmail.com

"These authors contributed equally to this work. post-harvest protection, especially in the upcoming harvest time. Awareness about the frequent residues and potential health hazards has promoted the demand for persistent and highly poisonous organic pesticides detection, ${ }^{7}$ particularly organochlorines (OCPs), organophosphates (OPPs) and pyrethroids (PYHs) in the plant-originated product.

Pesticide residues in the matrix are generally at a low level. Therefore, very sensitive, effective, and inexpensive methods established for broadly screening pesticides play an important role in the safe consumption of the plantoriginal product. When assessing advanced techniques for multi-pesticide analysis, including gas chromatographymass spectrometry (GC-MS), gas chromatography coupled to tandem mass spectrometry (GC-MS/MS), liquid chromatography coupled to tandem mass spectrometry (LC-MS/MS), and various biosensors, the affordability of gas chromatography with electron capture detection (GC-ECD) makes it the most attractive and universally employed in primary planting areas. ${ }^{8,9}$ Although GC-ECD has excellent sensitivity and reproducibility, analysis 
suffers as it requires adequate sample cleanup to exclude interference from complex matrices.

A variety of cleanup approaches are available for evaluating broadly contaminated multiclass pesticides in different matrices. Purification techniques, mainly solid-phase extraction (SPE), ${ }^{10,11}$ matrix solid-phase dispersion (MSPD), ${ }^{12,13}$ solid-phase microextraction (SPME),,$^{14,15}$ magnetic-solid phase extraction (M-SPE), ${ }^{16,17}$ and an improved quick, easy, cheap, effective, rugged and safe (QuEChERS) method, ${ }^{18,19}$ have been used on different samples. The most widely used pretreatment tool, SPE, can be automated. However, disposing of matrices with SPE columns is time-consuming and especially costly for pesticide analysis of multifunctional and combinations of herbal products. Although SPME and M-SPE eliminate cumbersome operations by integrating extraction, separation, and enrichment, SPME relies on specialized and selective micro-extraction fibers, making it expensive. In addition, both methods are alternatives with limited applicability in cases involving multiple classes of pesticides. To further enhance efficiency, some studies modified the cost-effective QuEChERS and tested packing excellent adsorbents into SPE tubes, separating most matrix from the targets using newly developed multiplug filtration cleanup (m-PFC) with assistance from a syringe. This method significantly simplifies pretreatment and seems to be feasible for the analysis of pesticides in fruits and vegetables, ${ }^{20,21}$ tomatoes, tomato sauce, ${ }^{22}$ and food samples. ${ }^{23}$ However, in most cases, multi-walled carbon nanotubes (MWCNTs) alone were applied to extract pesticides from foodstuff, the generally applicable solid-phase extraction sorbents including Cleanert PSA (primary secondary amine), Cleanert GCB (graphitized carbon black), Florisil, Alumina N, etc., have rarely been used in $\mathrm{m}-\mathrm{PFC}$. In addition, the cleanup requires a syringe together with an $\mathrm{m}-\mathrm{PFC}$ cartridge. In some complex matrices, such as herbal products, the capacity of m-PFC containing a single sorbent may not provide an effective enough cleanup for analysis.

Inspired by the techniques described above, we assembled a simple "push-and-pull" syringe filter containing the appropriate combination of packings, which consisted of various commonly used adsorbents, for efficient purification. In order to evaluate the feasibility of concurrently measuring multiple pesticides in complex products, we optimized and evaluated the effect of the amount of packing, frequency of push-and-pull, and elapsed time on this method. A total of 37 pesticides were measured in the 57 batches of purified Pericarpium Citri Reticulatae, where the levels of these pesticides were confirmed by GC-MS/MS. The cleanup method developed in this study proved to be fast, easy to operate, and highly efficient.
This method involving a syringe filter in combination with typically used adsorbents is expected to extend the evaluation of much more diverse matrices.

\section{Experimental}

\section{Chemicals and materials}

Standard solution of $100 \mu \mathrm{gL} \mathrm{mL}^{-1}$ of each pesticide and heptachlor epoxide (internal standard (IS)) were obtained from Agro-Environmental Protection Institute of Ministry of Agriculture (Tianjin, China). A mixed stock standard solution containing organochlorines $\left(2.0 \mu \mathrm{g} \mathrm{mL}^{-1}\right)$ and pyrethroids $\left(4.0 \mu \mathrm{g} \mathrm{mL}^{-1}\right)$ was freshly prepared by $n$-hexane. Due to the high toxicity of those pesticides, necessary precautions shall be taken in all operations. Acetone and $n$-hexane of HPLC grade were purchased from Thermo Fisher Scientific Co. Ltd. (Jiangsu, China). Adsorbents including Clearnert Florisil (150-250 $\mu \mathrm{m})$, Cleanert Alumina $\mathrm{N}(40-60 \mu \mathrm{m})$, Cleanert PestiCarb (GCB, 38-75 $\mu \mathrm{m})$, Cleanert $\mathrm{C}_{18}(50 \mu \mathrm{m}$, $60 \AA)$, Cleanert PSA $(40-60 \mu \mathrm{m})$, Cleanert $\mathrm{NH}_{2}(40-60 \mu \mathrm{m})$ and porous polypropylene plate $(20 \mu \mathrm{m})$ were purchased from Agela Technologies Co. Ltd. (Tianjin, China). $10 \mathrm{~mL}$ disposable syringes with polypropylene tube, piston and needle were purchased from Jiangsu Zhiyu Medical Instrument Co. Ltd. (Jiangsu, China).

\section{Sample preparation with needle filter}

Pericarpium Citri Reticulatae and its related products were collected from drugstores and herbal centers in China. The samples were dried and crushed into fine powder. $1 \mathrm{~g}$ of accurately weighed powder was immersed in $5 \mathrm{~mL}$ of a mixed solvent (hexane:acetone, $4: 1, \mathrm{v} / \mathrm{v}$ ), followed by vortex mixing for $1 \mathrm{~min}$, ultrasonic extraction for $5 \mathrm{~min}$ and centrifugation at $4000 \mathrm{rpm}$ for $5 \mathrm{~min}$. Then $2 \mathrm{~mL}$ of the supernatant was sucked into a pre-eluted needle filter consisting of GCB ( $0.5 \mathrm{~g})$, PSA ( $0.25 \mathrm{~g})$ and Florisil $(1.0 \mathrm{~g})$. The extract was dealt with four cycles of push-and-pull within 1 min using the needle filter and was concentrated to near dryness under nitrogen flow. Afterwards, the residues containing $0.2 \mu \mathrm{g} \mathrm{mL}^{-1}$ of internal standard was re-dissolved in $0.5 \mathrm{~mL}$ of $n$-hexane and centrifuged at $13000 \mathrm{rpm}$ for $10 \mathrm{~min}$, the prepared supernatant was transferred to sampler vial for following analysis.

\section{Analysis with GC-ECD and confirmation with GC-MS/MS}

The analysis was performed on an Agilent 6890 GC system equipped with electron capture detector and autosampler. Multi-pesticides were separated on an 
Agilent DB-1701 column $(30 \mathrm{~m} \times 0.25 \mathrm{~mm}, 0.25 \mu \mathrm{m})$ using a temperature program as follows: $120{ }^{\circ} \mathrm{C}$ (hold for $1 \mathrm{~min}$ ), ramp at $8{ }^{\circ} \mathrm{C} \mathrm{min}^{-1}$ to $180^{\circ} \mathrm{C}$ (hold for $2 \mathrm{~min}$ ), then ramp to $205{ }^{\circ} \mathrm{C}$ (hold for $6 \mathrm{~min}$ ) at $4{ }^{\circ} \mathrm{C} \mathrm{min}^{-1}$, increased to $270{ }^{\circ} \mathrm{C}$ at $5{ }^{\circ} \mathrm{C} \mathrm{min}^{-1}$, and finally ramp to $280{ }^{\circ} \mathrm{C}$ (hold for $10 \mathrm{~min}$ ) at $1{ }^{\circ} \mathrm{C} \mathrm{min}^{-1}$, with a gas flow of $1.0 \mathrm{~mL} \mathrm{~min}^{-1}$. Injection and detector temperature was held at 230 and $300^{\circ} \mathrm{C}$, respectively. The injection volume was $1 \mu \mathrm{L}$. The qualitative conformation was conducted on an Agilent 7890A GC system coupled to Agilent 7000A Triple Quadrupole GC/MS in electron impact ionization mode (EI, $70 \mathrm{eV}$ ). High purity helium (99.999\%) was employed as the carrier and quenching gas with a flow rate of $2.25 \mathrm{~mL} \mathrm{~min}^{-1}$, and nitrogen as collision gas was set at a flow rate of $1.5 \mathrm{~mL} \mathrm{~min}^{-1}$. The triple quadrupole was operated in multiple reaction monitoring (MRM) mode. The temperatures of the ion source and transfer line were 230 and $280{ }^{\circ} \mathrm{C}$, respectively. The MRM mode was operated for hexachlorobenzene $(283.8 / 248.8,283.8 / 213.9)$, p,p'-DDE (dichlorodiphenyldichloroethylene, 246.1/176.2, $315.8 / 246.0)$, procymidone $(139.0 / 111.0,251.0 / 139.0)$ and dicofol (282.8/96.0, 284.8/96.0).

\section{Results and Discussion}

\section{Syringe filter operating conditions}

The analytes in this study were primarily OCPs and PYHs, which have weak polarity and are relatively more volatile than other compounds. A mixture of $n$-hexane and acetone was prioritized as weak polar target and employed as extraction solvent. In view of the prevalence of volatile compounds in Pericarpium Citri Reticulatae, ${ }^{24}$ it was necessary to remove these co-extracts from the original solution firstly because they can interfere with targets and, thus, making detection of trace levels difficult and increasing the frequency of false positives. $\mathrm{m}-\mathrm{PFC}$ has received increasing attention due to its ease of operation and excellent purification ability. In this study, the syringe filter was assembled by placing a sieve plate at the bottom of a polypropylene column, loading the adsorbents into the column layer-by-layer, and then placing another sieve plate on top of the filter. The workflow of the assembled syringe filter is shown in Figure 1. A syringe filter facilitates the exclusion of interference by using larger amounts of various sorbents while recovering all of the extracted volume via rapid "pass-through" circulation. Related parameters, including types and quantities of adsorbents, duration of exposure, and the number of push-and-pulls, were assessed for their effects on and optimized for removing impurities and improving recovery.

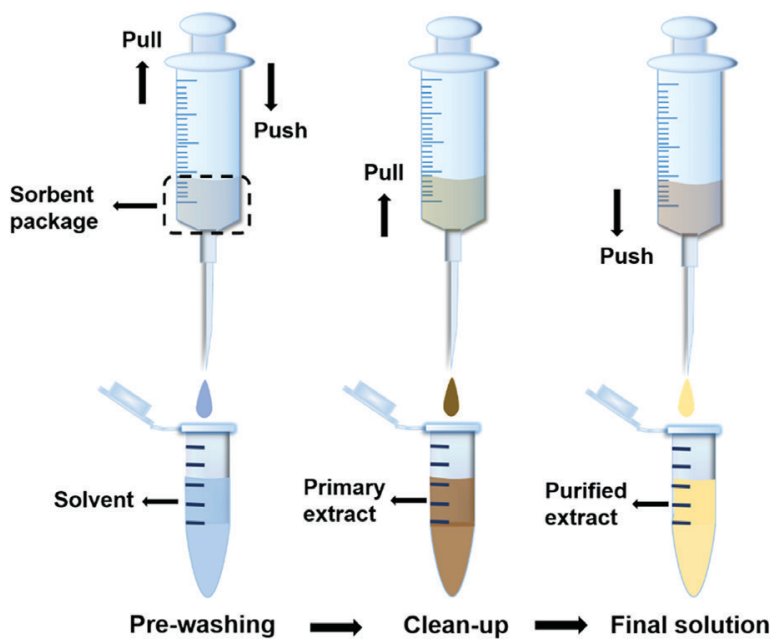

Figure 1. Schematic diagram of the rapid "push-and-pull" syringe filter based cleanup method.

\section{Selection of adsorbents}

The primary sample extract was firstly investigated, and the chromatogram was shown in Figure S1 (Supplementary Information (SI) section). A lot of co-extracted peaks interfere in the targets detection. Hence, appropriate sorbents should be used to remove the co-extracts from the sample solution. To identify the optimal packing materials, purification of a multipesticide complex co-extract was performed using readily available sorbents, including Florisil, Alumina N, GCB, $\mathrm{C}_{18}$, PSA, and $\mathrm{NH}_{2}$. As shown in Figure S2 (SI section), PSA and $\mathrm{NH}_{2}$ are effective for eliminating undesirable peaks with a retention time of less than $20 \mathrm{~min}$ in the gas chromatogram. Because they are rich in amino-groups, the adsorbent materials preferentially retain carbohydrates, organic acids, and fatty acids that were probably extracted from the Pericarpium Citri Reticulatae. There were more interfering peaks with high signal intensities at retention time of 30 min when using $\mathrm{NH}_{2}$ than PSA. As observed in the gas chromatogram between 30 and $50 \mathrm{~min}$. Florisil and GCB had higher sorbent capacities and better removal of interference compared to the other packings tested due to their excellent adsorption of pigments and steroids. Therefore, to remove the largest amount of co-extracts possible, a combination of GCB, PSA and Florisil was used in that order in the syringe column for the cleanup procedure. In addition, the quantity of the sorbents was optimized based on the recovery of 37 different pesticides, which yielded interesting results (Figure 2). A majority of the chlorinated analytes were beyond the scope of acceptable recovery rates (80-120\%) when an insufficient proportion of Florisil (GCB:PSA:Florisil, 1:1:1, $0.5 \mathrm{~g}$ ) was employed. The recovery rates for most of the 
pesticides tested fell below $100 \%$ when the proportion of PSA exceeded 50\% compared to GCB. The combination containing GCB:PSA:Florisil (2:1:4, 0.5:0.25:1.0 g) yielded desirable recovery rates for $86 \%$ of the target analytes. When large quantities of sorbents, in particular $\mathrm{GCB}$, were used for the matrix cleanup, the syringe filter retained large amounts of impurities and allowed the targets analytes to successfully pass through the filter, mainly as a result of repeated momentary exposure to these sorbents.

\section{Exposure time}

A proper duration of exposure to sorbents improved pretreatment efficiency. For 1 to $3 \mathrm{~min}$ of processing, the recovery rates met the requirements for most of the pesticides, except octachlorodipropyl ether (OCDPE), dicofol, $\beta$-cyfluthrin, and fenvalerate (Figure S3, SI section). A longer exposure led to enrichment of impurities in the filter, thus minimizing matrix interference. However, as the duration of exposure reached $3 \mathrm{~min}$, the yield of the targets of interest decreased to $60 \%$. Therefore, a 1 min exposure time was chosen to balance between increasing recovery and decreasing interference.

\section{Push-and-pull cycles}

The operation of syringe filter also could affect the exposure to sorbents, and the cycles of push-and-pull was evaluated accordingly. The spiked samples were separately operated at 3, 4 and 6 cycles, then recovery rates obtained were shown in Figure S4 (SI section). When the peel extract was purified using 3 cycles of push-and-pull, over half (51\%) of the analytes, including OCDPE, had a recovery of less than $80 \%$. This is likely a result of the balance of adsorption of analytes and interfering compounds by the sorbents. Unlike highly selective targeted molecules, these generally adsorptive materials have a weak affinity to some pesticides similar to interfering compounds. Therefore, the portion of the targets retained inside the sorbents resulted in a low recovery when 3 cycles were performed. A balance was reached at 4 cycles, where the majority of target analytes fell within the range of satisfactory recovery.

\section{Method validation}

\section{Specificity and matrix effect}

The specificity of the chromatography system could affect the accurate determination of the 37 pesticides of
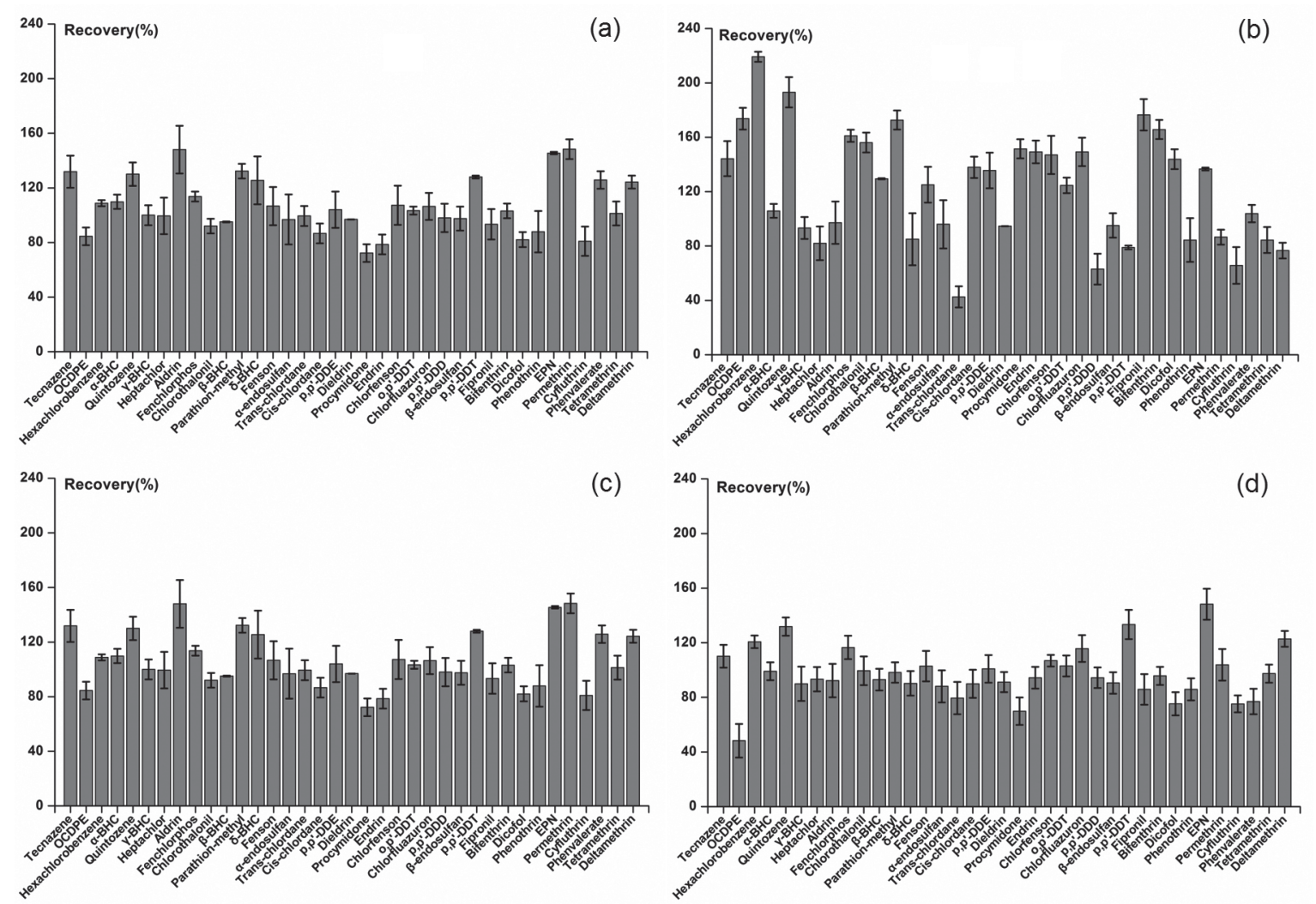

Figure 2. Comparison of recovery rates of spiked Pericarpium Citri Reticulatae solution at a concentration of $0.05 \mathrm{mg} \mathrm{kg}^{-1}(\mathrm{n}=3)$ by using package (a) GCB 0.25 g, PSA $0.25 \mathrm{~g}$ and Florisil $0.5 \mathrm{~g}$; (b) GCB $0.5 \mathrm{~g}$, PSA $0.5 \mathrm{~g}$ and Florisil $0.5 \mathrm{~g}$; (c) GCB $0.5 \mathrm{~g}$, PSA $0.25 \mathrm{~g}$ and Florisil $1.0 \mathrm{~g}$; (d) GCB $0.25 \mathrm{~g}$, PSA $0.5 \mathrm{~g}$ and Florisil $1.0 \mathrm{~g}$. 
interest. After purification using the optimized cleanup procedure, the extract turned from its original deep yellow color to clear when using the assembled filter, as shown in Figure 3. To examine the repeatability of the syringe filter, 3 samples were tested with the same filter in parallel after preparation (photos inset in Figure 3). Although the purified solutions of samples 2 and 3 appeared pale yellow due to the high adsorptive capacity of the packing, interfering impurities could be detected in the GC chromatograms (data not shown). Therefore, repeated use of the filter should be avoided to prevent cross-contamination and matrix interference, and to ensure accurate and reliable results. GC chromatograms are presented for blank solvent (Figure 3a), negative matrix solution (Figure 3b) and mixed standards (Figure 3c). Most of the pesticides in the chromatogram were cleanly separated with high selectivity and sensitivity. Chromatographic conditions and sample pretreatment effectively excluded impurities, preventing impurities from peel extract, solvent, and purified matrix from interfering with the analysis of the 37 pesticide residues of interest.

To assess whether the purified matrix had an effect on the signal intensity of the targets, the standards were separately prepared using pure solvent and blank matrix solution, at $0.8,0.04$, and $0.08 \mu \mathrm{gL}^{-1}$ (OPPs served as references). The matrix effect (ME) was calculated using the equation $\mathrm{ME}=\left(\mathrm{A}_{\mathrm{m}} / \mathrm{A}_{\mathrm{i}}\right) /\left(\mathrm{A}_{\mathrm{s}} / \mathrm{A}_{\mathrm{i}}\right)\left(\mathrm{A}_{\mathrm{s}}\right.$ is the peak area of pesticide in sample solution, $A_{i}$ is the peak area of internal standard, and $\mathrm{A}_{\mathrm{m}}$ is the peak area of pesticide in solvent). The results presented in Table 1 demonstrate that, except for p,p'-DDT (dichlorodiphenyltrichloroethane, 0.68), chlorothalonil (1.30), and bifenthrin (1.64), the MEs of the pesticides of interest ranged from 0.77 to 1.23 . According to the guidance SANTE/11945/2015, ${ }^{25}$ the criterion for ME is in the range of 0.8-1.2 and the MEs can be acceptable. These results indicate any effect the Pericarpium Citri Reticulatae matrix has on the analyte detection signal can be ignored after purification, as the proposed method had satisfactory specificity for the 37 pesticides assessed.

\section{Linearity and limits of quantification and detection}

To evaluate the performance of syringe filter pretreatment and GC-ECD analysis, a series of calibration standards were prepared in blank matrix extract. A matrix-matched calibration curve and internal standard were established for quantitative analysis. Table 1 summarizes the linearity, range, correlation coefficient (R), limits of detection (LOD), and quantification (LOQ). An adequate linear correlation fitting with $\mathrm{R}$ above 0.9971 was observed for the calibration curves. The LOD and LOQ values were obtained using stepwise dilution of standard solution with matrix solution, and the LOD and LOQ were considered as the levels with signal to noise ratio $(\mathrm{S} / \mathrm{N})$ approximating 3 and 10 . Under the optimized pretreatment and chromatographic conditions, concentrations as low as $0.375 \mu \mathrm{g} \mathrm{kg}^{-1}$ were detected, which is far below the default maximum residue limit of $10 \mu \mathrm{g} \mathrm{kg}^{-1}$ specified by the United States ${ }^{26}$ and European Union. ${ }^{27}$

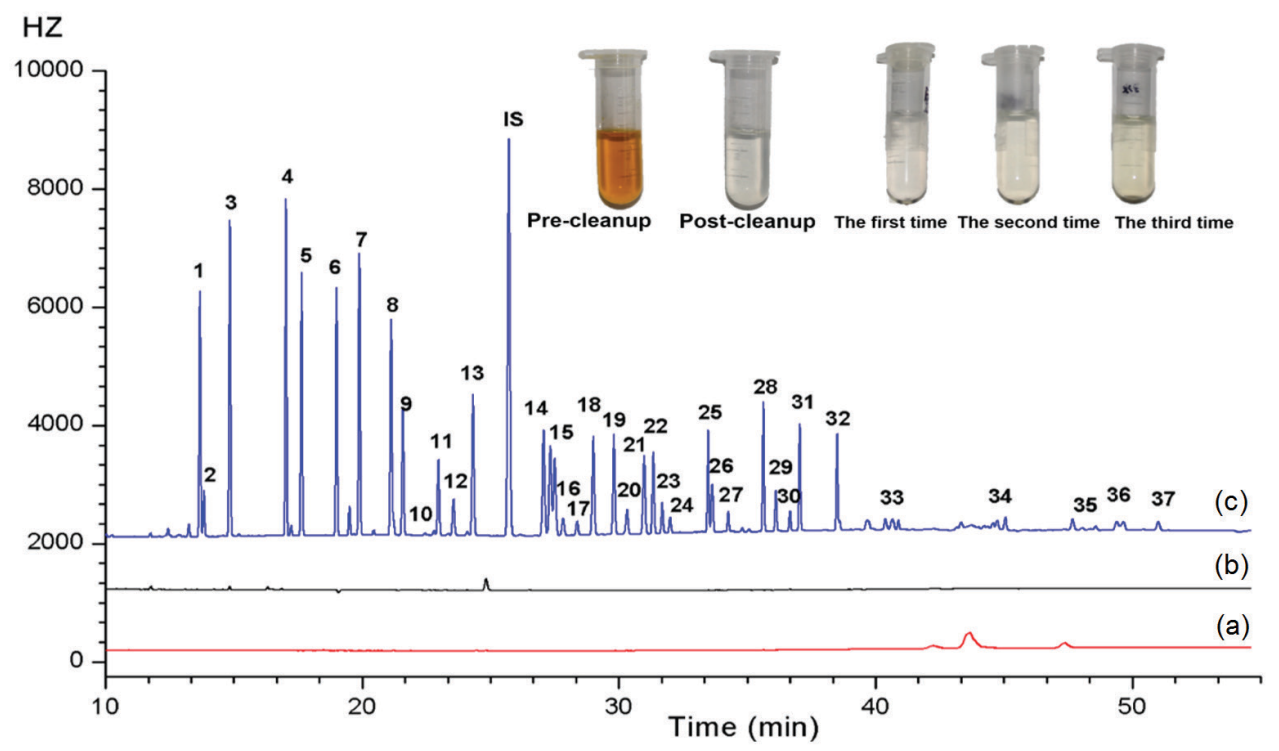

Figure 3. GC-ECD chromatograms of (a) blank solvent; (b) negative matrix solution and (c) mixed pesticide standards at $0.2 \mu \mathrm{g} \mathrm{mL}^{-1}$ (inset: photographs of the extract purified before and after by the needle filter and pretreated after three runs). 1: Tecnazene; 2: OCDPE; 3 : hexachlorobenzene; $4: \alpha$-BHC; 5: quintozene; 6: $\gamma$-BHC; 7: heptachlor; 8: aldrin; 9: fenchlorphos; 10: chlorothalonil; 11: $\beta$-BHC; 12: parathion-methyl; 13: $\delta$-BHC; 14: fenson; 15: $\alpha$-endosulfan; 16: trans-chlordane; 17: cis-chlordane; 18: p,p'-DDE; 19: dieldrin; 20: procymidone; 21: endrin; 22: chlorfenson; 23: o,p'-DDT; 24: chlorfluazuron; 25: p,p'-DDD; 26: $\beta$-endosulfan; 27: p,p'-DDT; 28: fipronil; 29: bifenthrin; 30: dicofol; 31: phenothrin; 32: EPN; 33: permethrin; 34: cyfluthrin; 35: fenvalerate; 36: tetramethrin; 37: deltamethrin; IS: heptachlor epoxide. 
Table 1. Matrix effect (ME), regression equations, linear ranges, correlation coefficients (R), limit of detection (LOD) and limit of quantification (LOQ) of 37 pesticides

\begin{tabular}{|c|c|c|c|c|c|c|}
\hline Pesticide & $\mathrm{ME}$ & $\mathrm{LOD} /\left(\mu \mathrm{g} \mathrm{kg}^{-1}\right)$ & $\mathrm{LOQ} /\left(\mu \mathrm{g} \mathrm{kg}^{-1}\right)$ & Regression equation & $\mathrm{R}$ & Linear range $/\left(\mathrm{mg} \mathrm{kg}^{-1}\right)$ \\
\hline Tecnazene & 1.03 & 0.375 & 1.25 & $y=0.0046 x-0.0041$ & 0.9999 & $0.00625-1.25$ \\
\hline OCDPE & 1.01 & 0.939 & 3.13 & $y=0.0007 x+0.0026$ & 0.9982 & $0.00625-1.25$ \\
\hline Hexachlorobenzene & 1.01 & 0.188 & 0.625 & $y=0.0058 x+0.0191$ & 0.9999 & $0.00625-1.25$ \\
\hline$\alpha-\mathrm{BHC}$ & 0.98 & 0.375 & 1.25 & $y=0.0063 x-0.0305$ & 0.9999 & $0.00625-1.25$ \\
\hline Quintozene & 1.04 & 0.188 & 0.625 & $y=0.0045 x+0.0149$ & 0.9998 & $0.00625-1.25$ \\
\hline$\gamma$-BHC & 0.99 & 0.375 & 1.25 & $y=0.0051 x-0.0387$ & 0.9998 & $0.0125-1.25$ \\
\hline Heptachlor & 1.09 & 0.375 & 1.25 & $y=0.0047 x+0.0008$ & 0.9995 & $0.00625-0.625$ \\
\hline Aldrin & 1.05 & 0.375 & 1.25 & $y=0.0058 x-0.0514$ & 0.9998 & $0.0125-1.25$ \\
\hline Fenchlorphos & 1.06 & 0.375 & 1.25 & $y=0.0026 x+0.0197$ & 0.9997 & $0.00625-1.25$ \\
\hline Chlorothalonil & 1.30 & 1.88 & 6.25 & $y=6 \mathrm{E}-05 x+0.0009$ & 0.9995 & $0.0125-1.25$ \\
\hline$\beta-\mathrm{BHC}$ & 1.10 & 0.938 & 3.125 & $y=0.0018 x+0.005$ & 0.9999 & $0.00625-1.25$ \\
\hline Parathion-methyl & 1.08 & 1.88 & 6.25 & $y=0.0008 x+0.0122$ & 0.9994 & $0.00625-1.25$ \\
\hline$\delta$-BHC & 0.99 & 0.375 & 1.25 & $y=0.004 x-0.0285$ & 0.9998 & $0.0125-1.25$ \\
\hline Fenson & 1.01 & 0.375 & 1.25 & $y=0.0029 x+0.0012$ & 0.9999 & $0.00625-1.25$ \\
\hline$\alpha$-Endosulfan & 1.00 & 0.375 & 1.25 & $y=0.0021 x+0.0002$ & 0.9998 & $0.00625-1.25$ \\
\hline trans-Chlordane & 1.05 & 0.375 & 1.25 & $y=0.0006 x+0.0006$ & 0.9999 & $0.00625-1.25$ \\
\hline cis-Chlordane & 1.03 & 0.375 & 1.25 & $y=0.0005 x+0.0016$ & 0.9999 & $0.00625-1.25$ \\
\hline $\mathrm{p}, \mathrm{p}$ '-DDE & 1.00 & 0.375 & 1.25 & $y=0.003 x-0.0097$ & 0.9999 & $0.00625-1.25$ \\
\hline Dieldrin & 1.23 & 0.375 & 1.25 & $y=0.0029 x-0.0028$ & 0.9999 & $0.00625-1.25$ \\
\hline Procymidone & 1.06 & 0.375 & 1.25 & $y=0.0006 x+0.0186$ & 0.9983 & $0.0125-1.25$ \\
\hline Endrin & 1.03 & 0.375 & 1.25 & $y=0.0023 x+0.0083$ & 0.9998 & $0.00625-1.25$ \\
\hline Chlorfenson & 1.11 & 0.375 & 1.25 & $y=0.0018 x+0.0071$ & 0.9999 & $0.00625-1.25$ \\
\hline o,p'-DDT & 1.16 & 0.375 & 1.25 & $y=0.0011 x-0.0296$ & 0.9980 & $0.0125-1.25$ \\
\hline Chlorfluazuron & 0.99 & 0.375 & 1.25 & $y=0.0004 x+0.004$ & 0.9997 & $0.00625-1.25$ \\
\hline p,p'-DDD & 1.04 & 1.88 & 6.25 & $y=0.002 x+0.0063$ & 0.9998 & $0.00625-1.25$ \\
\hline$\beta$-Endosulfan & 1.11 & 1.88 & 6.25 & $y=0.0009 x+0.0067$ & 0.9990 & $0.00625-1.25$ \\
\hline p,p'-DDT & 0.68 & 3.75 & 12.5 & $y=0.0007 x-0.0234$ & 0.9971 & $0.0125-1.25$ \\
\hline Fipronil & 1.02 & 1.88 & 6.25 & $y=0.0026 x-0.0095$ & 0.9999 & $0.00625-1.25$ \\
\hline Bifenthrin & 1.64 & 1.88 & 6.25 & $y=0.0008 x+0.0064$ & 0.9998 & $0.0125-2.5$ \\
\hline Dicofol & 1.03 & 1.88 & 6.25 & $y=0.0003 x+0.0063$ & 0.9986 & $0.00625-0.625$ \\
\hline Phenothrin & 0.77 & 1.88 & 6.25 & $y=0.0011 x+0.0008$ & 0.9999 & $0.0125-2.5$ \\
\hline EPN & 1.00 & 1.88 & 6.25 & $y=0.0015 x+0.0047$ & 0.9999 & $0.00625-1.25$ \\
\hline Permethrin & 0.99 & 3.75 & 12.5 & $y=8 \mathrm{E}-05 x+0.0028$ & 0.9990 & $0.0125-2.5$ \\
\hline Cyfluthrin & 1.05 & 3.75 & 12.5 & $y=0.0002 x-0.0014$ & 0.9995 & $0.0125-2.5$ \\
\hline Fenvalerate & 1.11 & 3.75 & 12.5 & $y=0.0002 x+0.0001$ & 0.9999 & $0.0125-2.5$ \\
\hline Tetramethrin & 1.10 & 3.75 & 12.5 & $y=1 \mathrm{E}-04 x+0.0003$ & 0.9998 & $0.025-2.5$ \\
\hline Deltamethrin & 1.05 & 3.75 & 12.5 & $y=0.0002 x-0.0031$ & 0.9995 & $0.025-2.5$ \\
\hline
\end{tabular}

OCDPE: octachlorodipropyl ether; BHC: benzene hexachloride; DDE: dichlorodiphenyldichloroethylene; DDT: dichlorodiphenyltrichloroethane; DDD: dichlorodiphenyldichloroethane; EPN: $O$-ethyl $O$-(4-nitrophenyl) phenylphosphonothioate.

\section{Precision, accuracy, and repeatability}

Depending on the analytical instruments used and sample preparation, the measurements of multiple pesticides will be affected by the precision, accuracy, and/or repeatability of the method. In this study, inter- and intra-day precisions were evaluated using $0.05 \mu \mathrm{g} \mathrm{mL}-1$ samples of OCPs and OPPs $\left(0.1 \mu \mathrm{g} \mathrm{mL}^{-1}\right.$ for PYHs $)$ using 6 continuous injections and 3 continuous days, respectively. Accuracy was measured with fortified samples containing all the pesticides at 3 known spiked levels (25, $\left.50,500 \mu \mathrm{g} \mathrm{kg}^{-1}\right)$. The fortified samples were prepared in advance, including adding the mixed standards to 
Pericarpium Citri Reticulatae, mixing well, and incubating for $30 \mathrm{~min}$. To validate the repeatability, 3 fortified samples for each level were processed and analyzed in parallel. As shown in Table S2 (SI section), GC-ECD analysis was highly precise with relative standard deviations (RSDs) in the range of $0.023-9.59 \%$ for inter-day and $1.31-11.9 \%$ for intra-day. The mean recoveries of most pesticides ranged between 70.9 and $128.6 \%$ with RSD values $\leq 19.1 \%$. Although the mean recoveries of hexachlorobenzene and p,p'-DDT were 69.2 and $66.3 \%$, the repeatability was acceptable with RSDs $\leq 8.1 \%$ and $\leq 5.7 \%$, respectively. Therefore, the analytical quality of the proposed method met the requirements for measuring trace pesticides in the complicated matrices.

\section{Pesticides residues in real samples}

The developed pretreatment and detection method was used to measure multiple pesticides in 57 batches of commercial Pericarpium Citri Reticulatae from China. Measurements using the internal standard method of the residues of the analytes in the samples are presented in Table S1 (SI section). Of the 37 types of pesticides, 4 OCPs, including hexachlorobenzene, p,p'-DDE, dicofol, and procymidone, were detected in 8 batches of commercial samples. In order to avoid false positives, the positive samples were confirmed by double characterized ion channel with GC-MS/MS (Figure 4).
Hexachlorobenzene and dicofol were the most frequently detected pesticides, where they were present in $10.5 \%$ of samples and the levels of hexachlorobenzene ranged from 0.03 to $0.2 \mathrm{mg} \mathrm{kg}^{-1}$. In addition, two batches exceeded the maximum residue limit $\left(0.1 \mathrm{mg} \mathrm{kg}^{-1}\right)$ for hexachlorobenzene set by USP 40, ${ }^{26}$ EP 9.0, ${ }^{27}$ BP 2017, ${ }^{28}$ and ChP 2015. ${ }^{29}$ This pesticide was widely used around the world until the last century and has a long period of activity in the environment. Long-term consumption of herbal product tainted with hexachlorobenzene likely increases the incidence of liver diseases from exposure to this chemical. Among the positive peels, dicofol residue was higher than the limit of $0.5 \mathrm{mg} \mathrm{kg}^{-1}$ in 4 samples. Because this substance can remain in plants for almost two years, this dicofol contamination deserves much attention. Both samples with procymidone had levels higher than the limits of $0.1 \mathrm{mg} \mathrm{kg}^{-1}$. Urgent measures are suggested to prevent public health issues as a result of dietary intake of polluted foodstuffs. Even though two batches of peels had detectable levels of p,p'-DDE, the residues were within the safe range. Overall, 5 of 57 batches were found to harbor persistent OCPs at levels higher than standards, in particular hexachlorobenzene, dicofol, and procymidone, where most peels contained more than two pesticides. The phenomenon was similar to a previous study, ${ }^{30}$ which showed a total of 17 pesticides detected in tangerine peel, Chinese matrimony vine, and jujube with more than $50 \%$ of the samples concurrently carrying at least two

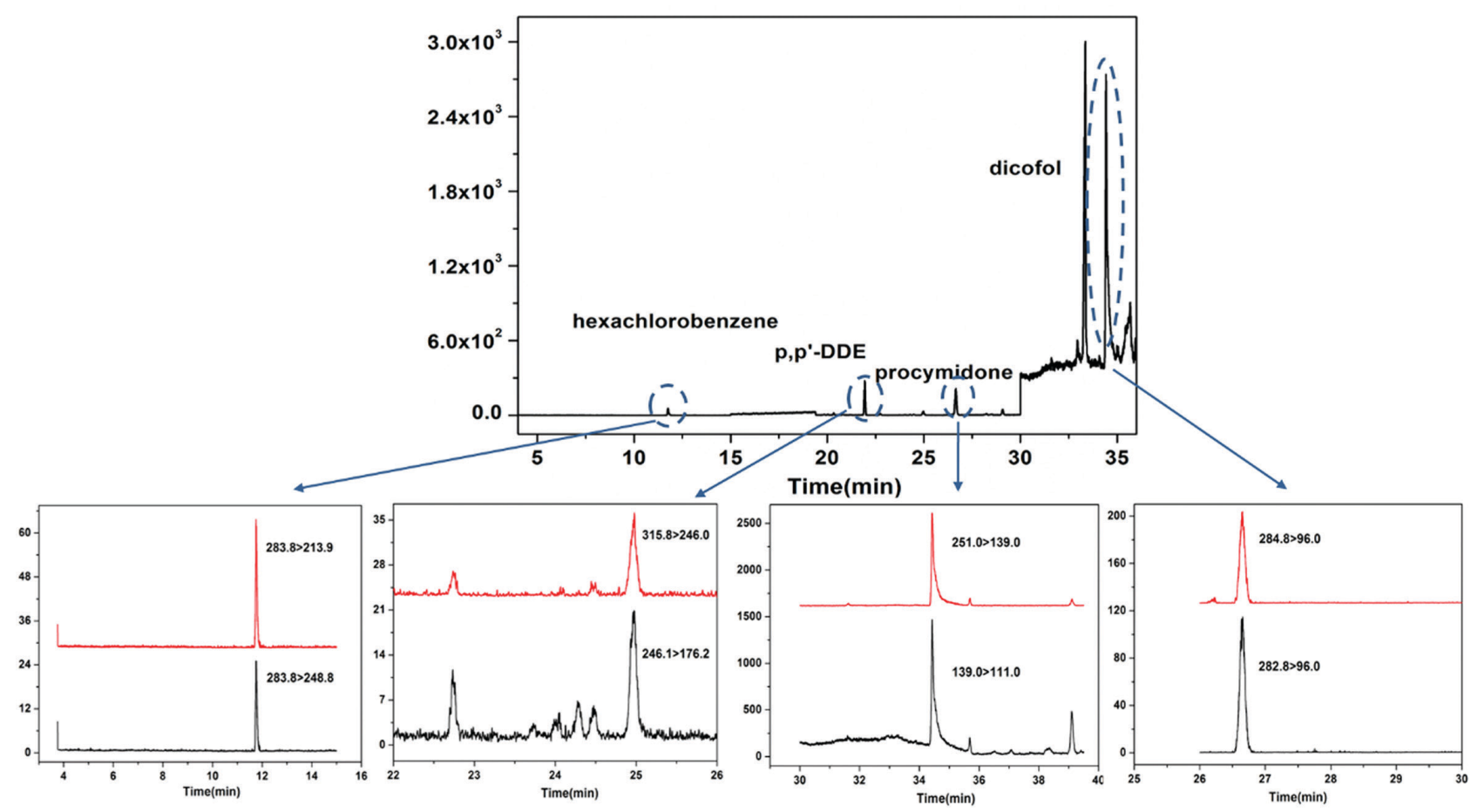

Figure 4. Representative total ion chromatogram of positive sample (S5) and selected ion chromatograms of hexachlorobenzene, p,p'-DDE, dicofol and procymidone. 
pesticides. Besides, Alves et al. ${ }^{31}$ detected residues of 4 organophosphates (OPPs) and 4 OCPs in commercial Brazilian citrus essential oils.

\section{Conclusions}

In this study, cleanup procedure and accurate GC-ECD analysis were combined with mass confirmation by GC-MS/MS to quantify the residues of persistent OCPs and high poison pesticides in Pericarpium Citri Reticulatae. The syringe filter was found to effectively minimize the matrix interference in Pericarpium Citri Reticulatae. Further work is being performed to evaluate the clean-up effect for multiresidues determination in other plant-originated herbs. When this technique was used to analyze real products, it detected only OCPs contaminating commercial samples. The most frequently detected OCPs were hexachlorobenzene and dicofol. Despite persistent OCPs being restricted or outlawed, these substances and several others still exceed the maximum limits in herbal products, suggesting these pollutants are probably derived from contaminated soil, water, and/or environment. In an attempt to ensure the safety of these plant-originated products, there should be significant enhancements in agricultural practices and supervision of these persistent pesticides.

\section{Supplementary Information}

Supplementary information is available free of charge at http://jbcs.sbq.org.br as PDF file.

\section{Acknowledgments}

This study was funded by National Natural Science Foundation of China (81573595, 81703699), CAMS Innovation Fund for Medical Sciences (No. 2016-I2M-1-012, 2016-I2M-3-010, 2017-I2M-1-013) and National Project for Standardization of Chinese Materia Medica (ZYBZH-Y-JIN-34).

\section{References}

1. Xu, J. J.; Wu, X.; Li, M. M.; Li, G. Q.; Yang, Y. T.; Luo, H. J.; Huang, W. H.; Chung, H. Y.; Ye, W. C.; Wang, G. C.; Li, Y. L.; J. Agric. Food. Chem. 2014, 62, 2182.

2. Hussain, A. I.; Anwar, F.; Sherazi, H. S. T.; Przybylski, R.; Food Chem. 2008, 108, 986.

3. Lu, M.; Yuan, B.; Zeng, M.; Chen, J.; Food Res. Int. 2011, 44, 530.

4. Santo, T.; Li, S.; Wang, R.; Iwasaki, S.; US pat. 76152392009 (JP02:086434).
5. Ortelli, D.; Edder, P.; Corvi, C.; Food Addit. Contam. 2005, 22 , 423.

6. Dou, X.; Chu, X.; Kong, W.; Yang, Y.; Yang, M.; RSC Adv. 2015, 5,86163 .

7. Du, J.; Gridneva, Z.; Gay, M. C. L.; Lai, C. T.; Trengove, R. D.; Hartmann, P. E.; Geddes, D. T.; Sci. Rep. 2016, 6, 38355.

8. Farina, Y.; Abdullah, M. P.; Bibi, N.; Khalik, W. M. A. W. M.; Food Chem. 2017, 224, 55.

9. Saini, M. K.; Mishra, S.; Alam, S.; Thakur, L. K.; Singh, O.; Raza, S. K.; Asian J. Res. Chem. 2016, 9, 1.

10. Hayward, D. G.; Wong, J. W.; Shi, F.; Zhang, K.; Lee, N. S.; DiBenedetto, A. L.; Hengel, M. J.; Anal. Chem. 2013, 85, 4686.

11. Mantzos, N.; Karakitsou, A.; Zioris, I.; Leneti, E.; Konstantinou, I.; Int. J. Environ. Anal. Chem. 2013, 93, 1566.

12. Chu, X.; Hu, X.; Yao, H.; J. Chromatogr. A 2005, 1063, 201.

13. Medina-Dzul, K.; Medina-Peralta, S.; Carrera-Figueiras, C.; Sánchez, M.; Muñoz-Rodríguez, D.; Int. J. Environ. Anal. Chem. 2017, 97, 831.

14. Huang, Z.; Chua, P. E.; Lee, H. K.; J. Chromatogr. A 2015 , $1399,8$.

15. Mehdinia, A.; Khani, H.; Mozaffari, S.; Microchim. Acta 2014, $181,89$.

16. Heidari, H.; Razmi, H.; Talanta 2012, 99, 13.

17. Jiang, C.; Sun, Y.; Yu, X.; Gao, Y.; Zhang, L.; Wang, Y.; Zhang, H.; Song, D.; Talanta 2013, 114, 167.

18. Lozano, A.; Rajski, Ł.; Belmonte-Valles, N.; Uclés, A.; Uclés, S.; Mezcua, M.; Fernández-Alba, A. R.; J. Chromatogr. A 2012, 1268, 109.

19. Paz, M.; Correia-Sá, L.; Vidal, C. B.; Becker, H.; Longhinotti, E.; Domingues, V. F.; Delerue-Matos, C.; J. Environ. Sci. Health, Part B 2017, 52, 48.

20. Zhao, P.; Fan, S.; Yu, C.; Zhang, J.; Pan, C.; J. Sep. Sci. 2013, 36, 3379.

21. Qin, Y.; Zhang, J.; He, Y.; Han, Y.; Zou, N.; Li, Y.; Chen, R.; Li, X.; Pan, C.; J. Agric. Food Chem. 2016, 64, 6082.

22. Zhao, P.; Huang, B.; Li, Y.; Han, Y.; Zou, N.; Gu, K.; Li, X.; Pan, C.; J. Agric. Food Chem. 2014, 62, 3710.

23. Qin, Y.; Zhao, P.; Fan, S.; Han, Y.; Li, Y.; Zou, N.; Song, S.; Zhang, Y.; Li, F.; Li, X.; Pan, C.; J. Chromatogr. A 2015, 1385, 1.

24. Hosni, K.; Zahed, N.; Chrif, R.; Abid, I.; Medfei, W.; Kallel, M.; Brahim, N. B.; Sebei, H.; Food Chem. 2010, 123, 1098.

25. SANTE/11945/2015: Method Validation and Quality Control Procedures for Pesticide Residues Analysis in Food and Feed, European Commission, 2015.

26. United States Pharmacopeia Convention; Articles of Botanical Origin; United States Pharmacopeia, 2016.

27. European Comission 2.8.13: Pesticide Residues, European Pharmacopeia, 2017.

28. British Pharmacopoeia Comission, Appendix XI L, Pesticide Residues, British Pharmacopoeia, 2017. 
29. Chinese Pharmacopoeia, General Rule 2341: Determination Method of Pesticide Residues, 2015.

30. Dou, X. W.; Chu, X. F.; Kong, W. J.; Yang, Y. H.; Yang, M. H.; RSC Adv. 2015, 5, 86163 .
31. Alves, A. A. R.; Rezende, M. J. C.; Hovell, A. M. C.; Bizzo, H. R.; Oliveira, A. C. L.; Rodrigues, S. V.; Rezende, C. M.; J. Braz. Chem. Soc. 2012, 23, 306.

Submitted: March 3, 2018

Published online: June 4, 2018 